\title{
FACTORES PERSONALES ASOCIADOS ALAISLAMIENTO SOCIOAFECTIVO, SOCIOEDUCATIVO, SOCIOECONÓMICO Y CULTURAL E IMPLICACIONES DEL CORONAVIRUS EN LA VIDA DE LOS SERES HUMANOS
}

PERSONAL FACTORS ASSOCIATED WITH ISOLATION, SOCIO-AFFECTIVE, SOCIO-EDUCATIONAL, SOCIOECONOMIC AND CULTURAL AND IMPLICATIONS OF THE CORONAVIRUS IN THE LIFE OF HUMAN BEINGS

\section{Elizabeth Soto Cortés}

\section{Elifeleth Soto Cortés}

\section{Socorro Clareth Olivo Álvarez}

Grupo de Investigación Malevajohu y Tepiche, Universidad de La Guajira

\footnotetext{
1 ELIZABETH SOTO CORTES

Correo.esotoc@uniguajira.edu.co

Institución. Universidad de La Guajira Estudios de pregrado Trabajador Social, Maestría en Educación.

Doctora en ciencias de la educación Código ORCID.0000-0002-3805-2768

Correo.esotoc@uniguajira.edu.co

2ELIFELETH SOTO CORTES

Correo.elisoto@uniguajira.edu.co Institución. Universidad de La Guajira

Estudios de pregrado. Administradora de empresas Postgrado. Magister en Diseño, Gestión y Dirección de Proyectos

Código ORCID. 000า-0002-3184-2767

3SOCORRO CLARETH OLIVO ALVARES

Correo.scolivo@uniguajira.edu.co

Institución. Universidad de La Guajira Estudios de pregrado. Administradora de empresas

Postgrado. Magister en Educación Código ORCID. 0000-0003-2023-7833.
} 


\section{RESUMEN}

La repercusión de la COVID 19 en la sociedad precisa el estudio de diversidad de factores, entre ellos los ambientales, los cuales hacen referencia al entorno o contexto donde se debe tener en cuenta la estimulación, la afectividad y las normas de crianza desde una edad temprana. Los factores culturales y socioeconómicos contemplan el desarrollo de cada niño, influenciado por el entorno social al que pertenece y la cultura de sus familias, valores sociales, religiosos, morales, éticos y estéticos. Cuando una persona ha vivido en sociedad y por razones extremas se encuentra privada de las relaciones con los demás, teniendo que vivir totalmente aislada, ésta asume su vida siguiendo los patrones que la sociedad le enseñó. En el subsistema de la sociedad se requiere la colaboración de todos sus miembros mediante una variedad de trabajos que hagan posible el desarrollo social y familiar para hacer más llevadero el aislamiento actual ocasionado por la pandemia. Este articulo toca las fibras de la sociedad guajira y sus raíces en las dimensiones socio afectiva, socioeducativa, socioeconómica y cultural, así como el rol familiar de la región.

\section{PALABRAS CLAVE: Aislamiento,} socioafectivo, socioeducativo, socioeconómico y cultural, Covid 19.

\section{SUMMARY}

Environmental factors, which refers to the environment of its context, where stimulation, affectivity must be taken into account; parenting standards, from an early age. Cultural and socioeconomic factors, where the development of each child is influenced by the social environment to which they belong, and the culture of their families, social, religious, moral, ethical and aesthetic values.

When a person has lived in society and, for these extreme reasons, they find themselves deprived of relationships with others, having to live totally isolated, they immediately begin to organize their life following the patterns that the society in which they lived taught them, in The subsystem of society requires the collaboration of all its members through a variety of works that make social and family development possible to make the isolation to which they are forced by the situation of the pandemic in which they live by Covid 19, in the second decade of the 21st century this article touches the fibers of Guajira society and its socio-affective, socio-educational socio-economic cultural roots and the family role of the region.

KEYWORDS: Isolation, socio-affective, socioeducational socio-economic, cultural Covid 19

\section{FACTORES PERSONALES ASOCIADOS}

\section{AL AISLAMIENTO}

La fase pandémica que se vive en los momentos actuales ha tenido un tremendo impacto, afectando aspectos de la vida de todos los seres humanos, tales como las actitudes, emociones y personalidad, entre otros (asociacioneducar. com, 2013). Se develan algunas características problémicas como la salud, el afecto, la compañía, el alimento, la educación y la economía, algo reiterativo a lo largo de nuestra existencia. De acuerdo con lo expresado se genera la siguiente interrogante. Cuál es el peso de la afectividad en a vida humana? Si la voluntad permite variar la conducta a través del aislamiento, ¿por qué muchas veces tan sólo se logra obtener poca felicidad cuando estamos acompañados, cayendo una y otra vez en el mismo ritmo de dependencia afectiva de donde deseamos salir?

En esta línea encontramos a (Larsen, 2005), para quien existen factores que inciden directamente en los aspectos biológicos, psicológicos, culturales, económicos y educativos que transforman el desenvolvimiento del individuo en la sociedad. Veamos. 
1) genética de la especie humana; reflejo de una sociedad dinámica que es capaz de evolucionar y adaptarse a los cambios afectivos, sociales, educativos y económicos.

2) genética individual o personal; donde cada quien busca tener una familia, sea nuclear, extensa, compuesta, monoparental o especial.

3) experiencias de vida propia; nos conduce a reflexionar sobre nuestras relaciones personales, que hacen exacerbamiento de factores como el estrés, mismo que genera en algunos casos un poco de violencia psicológica, física y baja autoestima con respecto a la salud mental en algunos miembros de la familia

4) experiencias de vida ajena; donde se recoge información sobre las interrelaciones familiares, maritales y donde convergen diversas dimensiones reflejadas en el siguiente gráfico.

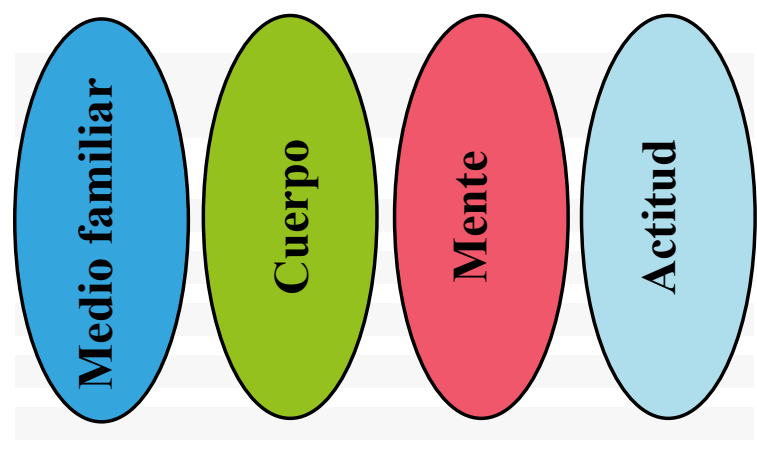

Fuente: Soto, Soto \& Olivo 2020

El medio familiar es el escenario por excelencia, que demanda mayor grado de afectividad. En este sentido (Aries, 2013) afirma que: "imitar la relación que tuvimos con nuestra madre o con nuestro padre, o la que ellos tenían entre sí es lo más natural en la dinámica familiar". Sobre todo cuando cada uno de los miembros ocupa su lugar y desempeña su función. De acuerdo con el autor en referencia:
Vale la pena pensar con qué frecuencia ese ambiente familiar que tuvimos, el cual se dio en un hogar propio, es una experiencia para replicar y si tratamos a nuestros hijos como nos trataban a nosotros con respeto, cariño, o si se escuchan las palabras "vamos a amarnos" y nos animamos de la misma forma como cuando éramos pequeños, cuando nos amaban y nos motivaban. ¿Cuántas veces se lo ha dicho usted a sí misma?

Eres maravillosa, te amo. O por el contrario en la corrección física solo se recibian regaños y castigos de la misma manera como lo hacían con nosotros

Es indiscutible el valor de las caricias emocionales en el ambiente familiar. Su práctica hace más llevaderos fenómenos como La COVID 19, que ha puesto al mundo en una situación de retroceso económico y filial con actividades que eran propias del siglo pasado, donde se convivía más tiempo con los miembros del núcleo primario, trayendo como consecuencia inadecuadas relaciones interpersonales $y$ violencia intrafamiliar; esto debido a que la vida de los seres humanos estaba desadaptada a una convivencia de mayor tiempo. Aquí la actitud juega un papel preponderante: la empatía, la asertividad, la comprensión, el amor y sobre todo la comunicación permitirán bajar el nivel de estrés causado por la rutina diaria que impide generar un mejor ánimo para seguir adelante. En este sentido lo ideal es la construcción de un buen ambiente familiar como eje fundamental demandado en esta época, incluyendo la articulación de cuerpo, mente y actitud, sobre todo en la orientación socioeducativa que ha sido una de las más afectadas por la pandemia. Como se sabe, a finales de 2019 se identificó en China un nuevo coronavirus al que se lo 
denominó COVID-19. Debido a su velocidad y la expansión y gravedad, el 11 de marzo-2020 la Organización Mundial de la Salud (OMS) lo declaró como pandemia impactando el sistema de vida mundial.

\section{5) Socioeducativo}

(Bertalanffy, 1928a, 1932, 1949a, 1960) expone en forma reiterada que: "Los lazos familiares se fortalecen a través de la comunicación para hacer que sus miembros sean más felices y extrañen menos el contexto en condiciones de normalidad o en ausencia de confinamiento". El análisis de los elementos actuales que hacen parte de la cotidianidad, enmarcado en La COVID-19, hace mención a las interrelaciones entre el docente y el estudiante mediadas por las TIC y TAC, asumiendo una experiencia poco transitada en la intervención con una herramienta tecnológica en donde el padre también asume el rol de orientador de los niños y niñas que se encuentran matriculados en las instituciones educativas, con lo que se impone una reducción significativa del juego con sus pares, generando en algunos de ellos tensiones y contradicciones sociales propias de una realidad que no alcanzan a comprender sobre todo aquellos de corta edad.

\section{6) culturales y familiares}

El quehacer de la familia se fundamenta en principios, valores y pautas de crianza influenciados directamente por la cultura. Sobre el particular (Cabrera-Gaytán, Vargas-Valerio, \& Grajales-Muñiz, 2014) consideran que:

La incidencia de la cultura en la familia muchas veces puede ser positiva o negativa, generando un crecimiento como ser humano para superar los instintos evolutivos $y$ que ellos no comanden nuestras vidas, ni afecten la de las personas con quienes convivimos. (p.438- 441).
En esta vía se asume como algo relevante desistir del pasado y disponerse a transformar las experiencias negativas recibidas en el sistema de vida desde pequeño, las cuales generan afectaciones psicomocionales y psicoculturales, entre otras. La COVID 19 impacta al núcleo familiar en la salud, la economía y también en la afectividad, por lo que se hace necesario realizar una intervención que permita trabajar aspectos como el autocuidado y medidas de seguridad en primera instancia, seguidos de encuentros con los ancestros e historias de vida que permitan identificar hechos que no se deben repetir y que en muchas ocasiones se deben desligar de actitudes cargadas de emociones negativas como la ira, el enojo, la tristeza y el miedo ejercidos en la familia como consecuencia de una práctica cultural, que con el fenómeno pandémico pueden causar serios problemas de salud mental.

\section{7) Cultura social}

La cultura social se percibe a través de comportamientos, expresiones y manifestaciones humanas que identifican a una sociedad. Dado que el sistema socio afectivo, socioeducativo y socioeconómico se encuentra afectado a nivel global con los nuevos retos que impone el fenómeno en referencia, el asistencialismo y aspectos como el paternalismo están llevando a las personas más pobres y de escasos recursos a actuar sin tener en cuenta las precauciones pertinentes para salir y evitar el contagio, violando en repetidas ocasiones los protocolos de seguridad; prima en muchos de ellos el hecho de percibir una ayuda por parte del estado, un subsidio, sea en efectivo o en especie, más que el cuidado de la salud física, mental y de la vida, y en esta circunstancia se hace más precaria su existencia.

\section{8) época histórica}

Hasta hoy han existido cinco etapas de la historia plenamente reconocidas; en los 
actuales momentos la historia se ha partido en dos con toda seguridad: el antes y el después de pandemia, pero nos ocupa es el momento actual donde este fenómeno afecta directamente al contexto familiar y trasciende el contagio hasta la cuarta generación en aquellas regiones donde el vínculo familiar tiene un significado de primer orden como es el caso de la región caribe, y en donde la práctica del afecto ágape a través de abrazos y besos contrarresta la de privación de las relaciones afectivas, educativas; el compartir con los padres, hijos, sobrinos, primos, nietos, hermanos hermanas y compadrazgo que hacen parte de la cotidianidad en el entorno Caribe demuestra la esencia comportamental de la familia nuclear, extensa, monoparentales, unipersonal o especiales debido a que siempre los demás familiares están inmersos en el día a día de cada uno de sus miembros haciéndose participes activos del diario vivir de sus familiares.

\section{9) Contextos ambientales particulares y culturales}

A nivel Global la problemática de la pandemia ha generado crisis insuperables en las diferentes dimensiones de las familias, el Departamento de La Guajira no es ajeno a esta situación. Para su gente, no ha sido fácil adaptarse al cierre del comercio, lo que deriva en ansiedad por recurrir a almacenes, por mejorar la presentación personal cuando al mirarse a un espejo se ve el desmejoramiento físico. Ha impactado también el estado de ánimo, por lo que es preciso advertir dos aristas de este problema: por un lado, el cierre de los establecimientos ha golpeado la economía negativamente tanto a los dueños como a los empleados; por otro, se afectaron los servicios de salud, se ha incrementado la demanda psicológica a causa de la baja autoestima por falta, entre otros aspectos, de verse bien, de sentirse bien debido a que laspersonas han pasado más tiempo en la casa donde en algunos casos las parejas se comentan "hoy te ves como rara" por no decir "cómo te hace falta ese arreglo personal", que de una u otra forma es violencia verbal, lo que genera un choque emocional en cada uno de los miembros.

Estas condiciones de aislamiento afectan también lo conductual, afectivo, educativo, el desarrollo social entre pares, hermanos, hijos, padres. Así mismo, se agrega la información masiva del terrorismo de información sobre la situación actual y el exageramiento por parte de quienes tienen credos religiosos donde se aprovechan para hacerle daño psicológico a muchas personas con ideas absurdas, forjando un debilitamiento en el rol familiar, frente a lo cual se sugiere buscar apoyo profesional principalmente en aquellos que desempeñan roles laborales como es el caso de médicos, paramédicos y enfermeras o conductores de ambulancia y de taxis, al igual que servidores de la fuerza pública para que atiendan aquellos casos donde las personas se encuentran en condiciones de vulnerabilidad emocional pero también supliendo las necesidades básicas insatisfechas a través de la solidaridad, compañerismo y familiaridad, en razón de salvaguardar las tradiciones ancestrales que venían en decadencia.

\section{Personales}

(Oliver, 2020). "Presentan las características de la muestra que tienen que ver con la situación personal con respecto a la pandemia personas había experimentado diversos temores y preocupaciones, los temores más recurrentes fueron a que se contagiara alguna persona" (p.10-14).

En el aspecto personal es necesario fortalecer en este estadio de la pandemia de La COVID -19 el autoreconocimiento como medio para identificar debilidades y fortalezas que conduzcan a vivir un proyecto de vida acorde con las exigencias 
actuales y que se encuentren centrados en la familia construyendo los procesos que conducen a ser mejores seres humanos, útiles para una sociedad dinámica como la presente.

\section{LA PAZ COMO EFECTO EMOCIONAL Y PERSONAL}

Las experiencias de proyectos de vida individual y familiar se están llenando de dolor a causa del maltrato generado en una convivencia desprovista de Educación emocional. Para (Arango, 2009). Considerar que: "Si tratamos a las personas con la misma consideración y respeto con el que nos gustaría ser tratados, mejoraríamos sustancialmente su calidad de vida y de paso la nuestra, pues evitaríamos conflictos innecesarios que lo único que hacen es desgastarnos física, psicológica y moralmente". (p.11). En estos tiempos de pandemia se aumenta cada día la incertidumbre, el sentimiento de impotencia, resignación, desconcierto, al querer abrazar la paz interior pero también la paz estable y duradera que tanto pregona el estado colombiano en sus políticas de estado.

En esta dirección la educación ha de responder a estas exigencias. Se requiere producir cambios de comportamiento en estos tiempos de confinamiento en que aumentan las exigencias en el manejo del sistema emocional, pues se debe integrar las competencias sociales de la educación para la vida para disminuir un poco la violencia que se vive en los diferentes medios donde se desenvuelven las personas. Ello implica perfeccionar nuevas competencias personales como es la tolerancia, la asertividad, la ternura, el comprender la empatía y asertividad y sobre todo la aceptación del otro. Un componente emocional estable permite una mayor integración en el aula de clase y en la vida familiar y comunitaria.

\section{MOTIVACIÓN}

Para (Goleman D. , 2020). “La motivación hace parte del impulso del logro movido por recompensas externas". (p.460). Según él, la primera señal es una pasión por el trabajo mismo en busca de una recompensa. La segunda es el despliegue de una energía para hacer mejor las cosas cada día, por lo que las personas con una alta motivación permanecen optimistas, aún cuando la calificación del desempeño esté en su contra. Para tal fin, la autorregulación concierta con la motivación para sobrellevar la frustración y la depresión que aparece después de un fracaso o un retroceso, eventos muy comunes en este confinamiento. La alta motivación y el optimismo han representado antídotos naturales, que junto con las infusiones de medicina natural han aportado en la vida de los menos favorecidos .

\section{Inteligencia y aptitudes}

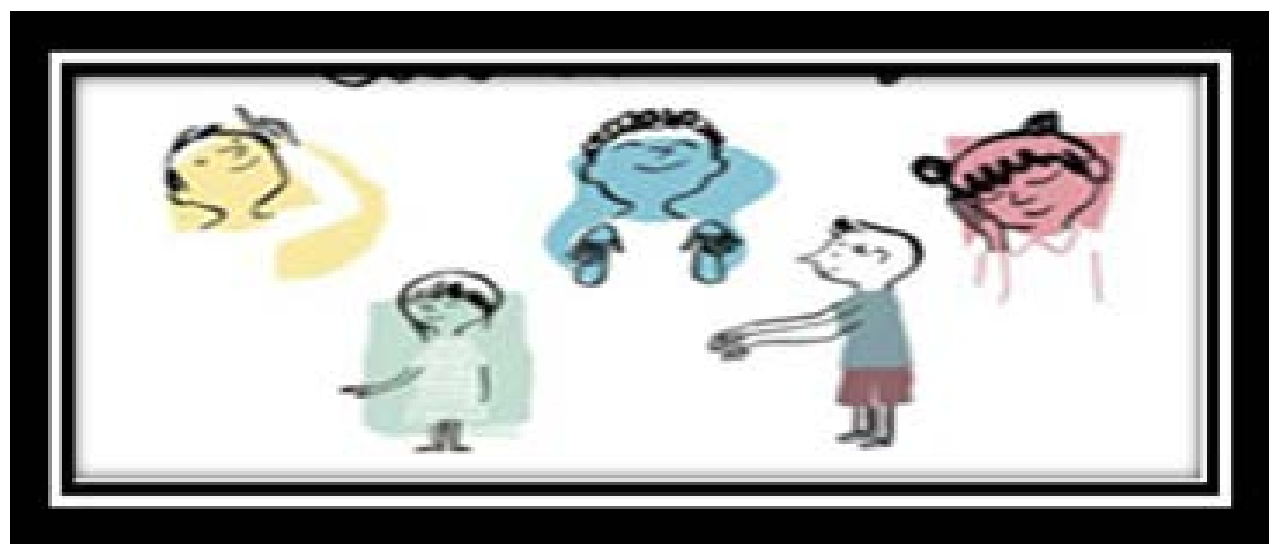

Fuente: Google Académico 2020. 
Son muchas las definiciones que se le ha concedido a la inteligencia a lo largo del tiempo se han desarrollado diferentes perspectivas; produciéndose ciertos avances que fueron haciendo posibles los desarrollos posteriores; anotaremos algunas de donde se desprende un estudio de referencias bibliográficas digital de archivos y otros documentos que arrojan las variables, dimensiones e indicadores de ésta investigación, además, su caracterización y estrategias para alcanzarlas, a partir de esta información se determinó la influencia de la enseñanza aprendizaje educativo para el desarrollo de la inteligencia emocional.

La palabra inteligencia proviene del latín "Intelligentia" y puede interpretarse según la real academia española. Real Academia Española. (2001). así:

- Capacidad de entender o comprender.

- Capacidad de resolver problemas.

- Conocimiento, comprensión, acto de entender.

- Habilidad, destreza y experiencia.

- Trato y correspondencia secreta de dos o más personas o naciones entre sí

- Sustancia puramente espiritual.

En el contexto occidental la noción de inteligencia se vincula con el desempeño de actividades útiles, con la aparición de fábricas e industrias el significado de inteligencia quedó ligado al método de enseñanza, pasando de la repetición de tareas concretas y la transmisión oral, al dominio de la ciencia y la cultura; se considera como una cualidad individual que permanece constante a lo largo de la vida y que puede ser medida de forma continua realizando comparaciones respecto a otras personas o grupos de referencia. (FUNIBER, 2015)

No se puede desconocer el pensamiento de "Una escuela que educa para la comprensión es aquella que promueve el aprendizaje significativo y la transferencia del conocimiento a partir de diferentes formas o maneras de acceder a él, considerando más importante la calidad de los aprendizajes que la calidad de los conceptos que se aprenden (Gardner, 2013).
Donde propone un concepto completamente nuevo que aún hoy día sigue siendo innovador frente a la idea imperante de que existe una única inteligencia medible como la que en su día desarrollo Binet un potencial biosociológico para procesar información que se puede activar en un marco cultural para resolver problemas o crear productos que tienen valor para una cultura"(p.177).

Esta teoría le permite a los seres humanos transformar las actividades para que se vean normales, por una experiencia multisensorial para todos; donde debe identificar o descubrir las inteligencias fuertes o destacables en cada uno, para que a través de ellas se puedan potencializar las otras en este caso son los miembros de las familias quienes asumen los roles de orientadores escolares puesto que la virtualidad educativa es una nueva etapa que viven los hijos donde hay que entrar a explicar por qué no se puede salir a la escuela en forma presencial.

En lo que actitud se refiere la sabiduría ancestral de los Pueblos Indígenas, propios del caribe Colombiano con los mayores sabedores frente a la situación actual de la pandemia y otras desarmonías que presentan los territorios indígenas apuntan a que es necesario aprender a saber cómo actuar ante la incertidumbre para no dejarse llevar por noticias falsas o el pánico.

Por otro lado, está el aspecto relevante de la inteligencia y actitudes que se ven reflejadas en el pueblo Guajiro como son los sentimientos de miedo, angustia, dolor y ansiedad junto con sentimientos de responsabilidad, cuidados 
y valorización de la interdependencia, afecto, la posibilidad de introspección y reflexión de la pandemia que se generarían al interior de las familias, es aquí donde el manejo de la inteligencia emocional en conjunto con la actitud hace más llevadera la situación de confinamiento y de aislamiento que se ha dado alrededor del contexto donde se perciben las diferencias sociales y de género en la población, respecto al nivel educativo y el confort percibido durante el aislamiento social, constituyen indicadores que dan cuenta la desigualdad de recursos culturales y materiales en el marco de la crisis sanitaria y especialmente en el impacto mental que provoca la pandemia.

Permitiendo un equilibrio en la parte emocional. Cabe resaltar que esta va muy de la mano con la actitud que se asume al interior del individuo como ser humano sentí pensante.

\section{EMOCIONALES}

Las competencias emocionales de las personas deben estar en óptimas condiciones, sobre todo en estos tiempos de confinamiento por $\mathrm{La}$ COVID-19 propician el manejo de las emociones "negativas" para relacionarse con el otro y convertirlas en emociones "positivas". En esta vía (Morín, 2011) es claro:

"La educación del futuro debe ser una enseñanza universal centrada en la esencia humana, para comprender el conocimiento de las partes, del origen $y$ el contexto dentro del Cosmos y los elementos y relaciones materiales que lo construyen"(p.75).

En este orden de ideas la Inteligencia emocional se relaciona con el aprendizaje en una forma directamente proporcional, es decir a mayor inteligencia emocional, mejor rendimiento académico, pues las relaciones interpersonales permeadas por un trato inteligente contribuyen al equilibrio emocional y a la obtención de un buen rendimiento académico acompañado de adecudas relaciones sociales. Educar en las emociones es un reto inobjetabe de la educación, para que sirva finalidades formativas. Para decirlo con Arboleda ${ }^{1}$, como proceso edificador la educación es del tamaño del potencial de educar. El acto de educar no espera, se expresa en las oportunidades y capacidades mínimas que los miembros de las instituciones sociales (escuela, iglesia, familia, medios de comunicación, entre otros) generen para intervenir en la formación (léase personalización/ humanización) del otro y de sí mismo.

Para (Morín, 2011):

Sólo un pensamiento capaz de captar la complejidad de nuestras vidas, nuestros destinos y la relación individuo/ sociedad/especie, junto con la de la era planetaria, puede intentar establecer un diagnóstico del curso actual de nuestro devenir, y definir las reformas vitalmente necesarias para cambiar de vía.

Sólo un pensamiento complejo puede darnos armas para preparar la metamorfosis social, individual $y$ antropológica (p. 29).

Nos acerca sin lugar a dudas hacia lo que se requiere en la sociedad actual y futura acerca de las interacciones entre los objetos, las personas y el ambiente como un todo; entonces el rol de la familia, y de las autoridades educativas, de los medios de comunicación, del sector productivo, debe abrazar una nueva forma de pensar que conduzca a un mejor bienestar emocional.

(Ekman, 2011). Afirma que: "hay emociones centrales que se observan en cuatro expresiones faciales: el miedo, la ira, la tristeza y la alegría. Estas expresiones son reconocidas por diversas

$1 \quad$ Arboleda JC. Formación para la vida: de las competencias a la comprensión edificadora. bol.redipe [Internet]. 27 de diciembre de 2017 Disponible en: https://revista. redipe.org/index.php/1/article/view/300 
culturas hechos considerados universales". (p.62.) Los países tercermundistas no están preparados para esta pandemia de la COVID-19 mucho menos el departamento de La Guajira que tiene unas características especiales desde lo cultural hasta el sistema educativo y familiar.

10) Calidad de la nutrición alimenticia recibida desde lo psicoafectivo.

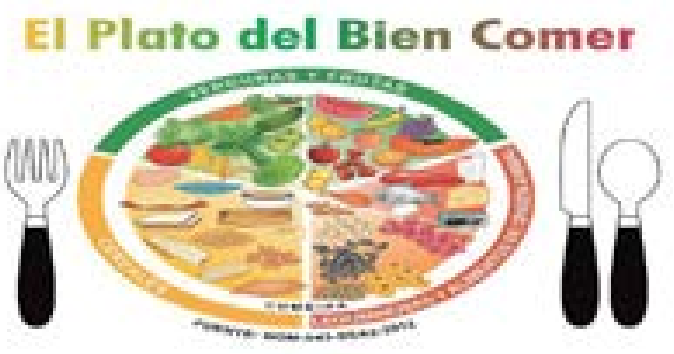

Fuente: Google Académico 2020

Durante esta etapa de confinamiento por La COVID-19 no hubo un término específico que conceptualizara las características de la "alimentación correcta". Para (Suárez Solana, 2016), se siguió utilizando el de "dieta normal"; sin embargo, hubo cambios en el significado, ya que no se priorizó tanto, como en la etapa anterior, el consumo de proteínas de origen animal y se dio mayor importancia a la satisfacción de necesidades individuales de nutrimentos orgánicos (vitaminas y minerales) compuesto por cereales, proteínas, frutales, legumbres y tubérculos lo cuales hacen parte de la dieta diaria de las familias del Caribe y más concretamente en la península de La Guajira quienes se han visto afectados por los centros que prestan el servicios alimenticios; caso concreto que podemos anotar: donde se encuentran la tradicional arepa de queso que puede ser usada como desayuno o cena y que no solo cumple la función de tomar los alimentos sino desde lo que representa social y afectivamente dado a que cada espacio de estas ventas sirven como zonas de socialización de la familia y las amistades generando un vínculo de afectividad al compartir con el otro . Si se analizan los suministros de la plaza de mercado se ve la ausencia del tradicional friche hecho de carne caprina o chivo el cual es otro espacio de comunicación y socialización filial y familiar extrañado en gran manera, pues es un espacio donde se comparte con la cultura propia autóctona de la región Guajira

Con este aislamiento se ve la brecha que ha traído como consecuencias la pandemia que no solo afectó la parte alimentaria y nutricional sino factores personales asociados al aislamiento, socio afectivo, socioeducativo socioeconómicos cultural en la vida de seres humanos en el contexto Guajiro.

\section{1) Implicaciones del Coronavirus en la Vida de Seres Humanos}

Sin emitir juicios de valoración a priori nuestra cultura posee la capacidad para sobrevivir o adaptarse a este mundo, pues la renovación de los conocimientos es cada vez más acelerada que en cualquier época que se haya vivido en el contexto. Se puede decir que ha generado lo que se conoce como fatiga pandémica, ocasionando situaciones de estrés, irritabilidad y falta de esperanza, dificultando el buen funcionamiento de la vida propia y de nuestro prójimo. Algo que nos hace perpetuar más el problema de la pandemia es el estrés, que puede finalmente incidir en la vida de muchas personas viéndose afectadas con la pandemia de La COVID -19, que luego a su vez seguirán con esta cadena invisible de transmisión perturbando la salud mental de las comunidades sobre todo Guajira. Es preciso anotar que: esta cultura es muy dependiente psicológica y afectivamente de su familia, buscando asegurar la supervivencia y la transcendencia sin perder la esencia de sus ancestros a partir de la práctica del aislamiento, socio afectivo, socioeducativo socioeconómicos 
cultural por las implicaciones del coronavirus se pone en riesgo toda esa riqueza cultural.

(Agency., 2013). En la vida de seres humanos. Manifiesta que: Las tres soluciones posibles que están a nuestro alcance para resolver el problema humano son:

1) Esperar a que la evolución de la pandemia se dé de la mejor manera y nuestras familias salgan lo mejor libradas y haya menos contacto físico con familiares y amistades.

2) Para (Trenas, 2012)“Los padres e hijos deben ser más disciplinados en la interacción diaria con los hijos". (p.12). Esto con la finalidad de sobrellevar el aislamiento donde se realizan las tareas en un tiempo más corto o más llevadero a través de la tecnología pues los padres asumen el papel de orientadores de las actividades académicas más el acompañamiento de los docentes que están dirigiendo los procesos escolares.

3) Una educación dirigida a conocer, comprender, controlar, modelar y supervisar nuestro mundo interior de forma consciente para las nuevas generaciones millennials' que también son miembro de nuestra cultura.

A manera de conclusión, una relación inextricable entre los factores personales asociados al aislamiento socio afectivo, socioeducativo, socioeconómico y cultural y las implicaciones de La COVID-19 en la Vida de los Seres Humanos, que ha logrado impactar en los sistemas de adaptación en los diferentes contextos.

\section{CONCLUSIÓN}

Se ha abordado algunos de los factores personales asociados al aislamiento socioafectivo, socioeducativo, socioeconómico y cultural, así como las Implicaciones
COVID-19 en la vida de las personas. El mundo de hoy presenta desafíos que cada vez más complejos para nuestra sociedad, siendo el tiempo propicio para enfocarse en actividades que pueden fortalecer los usos y costumbres de nuestros ancestros, y además hacer en el hogar aquellas actividades que disminuyan el estrés por el confinamiento como cultivo de pan coger, jardinería o manualidades como tejer. La cuarentena es buen momento para aprender algo nuevo o realizar actividades pendientes como practicar un instrumento musical, la terapia del perdón, o simplemente reflexionar; la inteligencia emocional es de gran ayuda porque evita caer en estrés o pánico.

La pandemia de La COVID-19 está afectando el sistema de una sociedad, como es el caso de las familias en sus aspectos psicoafectivo, socioeducativo y socioeconómico, entre otros. En lo socioeducativo la tecnología se ha convertido en el medio de comunicación predominante aun para aquellas culturas en vías de desarrollo; también los modelos de vida presentes en cada cultura cuyas estructuras han sido modificadas buscando formas $y$ capacidad de adaptación, asumiendo nuevos retos. Tenemos la esperanza de sobrevivir con las reglas impuestas para mejorar la existencia de los seres humanos. Podemos en el Caribe Colombiano aplicar la sabiduría propia y el conocimiento occidental para no ser afectados por la pandemias; sin embargo, son muchas las vidas que se han desprendido del seno de muchas familias vencidas por esta pandemia: Así lo confirma (Ángel, 2014): "Afecta el entorno familiar social y económico, trae la angustia o estrés entristeciendo los lazos familiares grupales". En este orden de ideas, el contexto guajiro se mantiene pese a las dificultades con el esfuerzo de las generaciones presentes y del futuro, para que cada vez este poblado, con esta mayor cantidad de personas fortalecidas y con sentido común, pueda paso a paso revertir 
la situación actual y poner de nuevo a nuestro mundo en la posición adecuada para todos.

\section{REFERENCIAS BIBLIOGRÁFICAS}

Agency., T. H. (2013). The Health Protection Agency. UK Novel Coronavirus Investigation team. Evidence of personto-person transmission within a family cluster of novel coronavirus infections, United Kingdom. UK Novel Coronavirus Investigation team. Evidence of personto-person transmission within a family cluster of novel coronavirus infections, United Kingdom, February (pág. Surveill. ). Euro Surveill. 2013;18(11):pii=20427.: The Health Protection AgencyThe Health Protection Agency. United Kingdom, February 2013. Euro Surveill. 2013;18(11).

Ángel, P. M. (2014). Manual de investigación en medicina Escuela de Medicina de la Universidad de Celaya Revisado en cuanto a metodología por Dr. Roberto Hernández Sampieri Junio, 2014Infecciosas de EE UU ha compartido las imágenes d. En P. H. Ángel, Manual de investigación en medicina Escuela de Medicina de la Universidad de Celaya Revisado en cuanto a metodología por Dr. Roberto Hernández Sampieri Infecciosas de EE UU ha compartido las imágenes del COVID19 (págs. 36-49). EE UU: Manual de investigación en medicina Escuela de Medicina de la Universidad de Celaya Revisado en cuanto a metodología por Dr. Roberto Hernández Sampieri Junio,.

Arango, L. E. (2009). Inteligencia Emocional. Aprendiendo y creciendo juntos:. Bogotá (Colombia).: Ediciones Gamma S.A.
Arboleda JC. Formación para la vida: de las competencias a la comprensión edificadora. bol.redipe [Internet]. 27 de diciembre de 2017 Disponible en: https://revista.redipe.org/index. php/1/article/view/300

Aries, P. (2013). El niño y la vida familiar en el Antiguo Régimen. Madrid: Editorial Taurus.

asociacioneducar.com, A. E. (2013). Asociación Educar Ciencias y Neurociencias Aplicadas al Desarrollo Humano. Argentina: Impreso en Argentina ISBN 978-987-26988-0-5.

Bertalanffy, L. v. (1928a, 1932, 1949a, 1960). Teoría de sistemas. Cuyas ideas no salían de entre las cubiertas de los libros-están creando un mundo nuevo., Recuperado en http://suang. com. ar 32.

Cabrera-Gaytán, D. A., Vargas-Valerio, A., \& Grajales-Muñiz. (Cabrera-Gaytán, David Alejandro; Vargas-Valerio, Alfredo; Grajales-Muñiz, Concepción Infección del nuevo coronavirus: nuevos retos, nuevos legados Revista Médica del Instituto Mexicano del Seguro Social, vol. 52, núm. 4, 2014, pp. 438441 Instituto Mexic de Cabrera-Gaytán, David Alejandro; Vargas-Valerio, Alfredo; Grajales-Muñiz, Concepción Infección del nuevo coronavirus: nuevos retos, nuevos legados Revista Médica del Instituto Mexicano del Seguro Social, vol. 52, núm. 4, 2014, pp. 438- 441 Instituto Mexic de 2014). Concepción Infección del nuevo coronavirus. Concepción Infección del nuevo coronavirus: nuevos retos, nuevos legado (págs. 438- 441 ). Mexico: Revista Médica del Instituto Mexicano del Seguro Social, vol. 52, 
núm. 4, 2014, pp. 438- 441 Instituto Mexic.

Ekman, P. (2011). Handbook of Cognition and Emotion (pp. 45-60). New York: John Wiley y Sons Ltd. Basic Emotions (Eds.), Handbook of Cognition and Emotion, 45-60.

FUNIBER. (2015). Fundación Universitaria Iberoamerciana de Estudios Superiores . Fundación Universitaria Iberoamerciana de Estudios Superiores , 15-20.

Gardner, H. (2013). La inteligencia reformulada: Las inteligencias múltiples en el siglo XXI. Madrid: Paidos Iberica.

Goleman, D. (2020). La inteligencia emocional se puede mejorar y aprender. En G. Daniel, Cinco aprendizajes sobre la Inteligencia Emociona a. La inteligencia emocional se puede mejorar y aprender (pág. 177). Wastom USA: Editorial Kairos.

Goleman, D., \& Boyatzis, R. y. (2004). El Líder Resonante Crea Más. El Poder de la Inteligencia Emocional. . Venezuela.: Editorial Melvin C.A. pp. 351.

Larsen, R. y. (2005 ). Psicología de la personalidad. Dominio del conocimiento sobre la naturaleza humana. . México : Mc Graw Hill Interamericana. pp. 651. .

Morín, E. E. (2011). Seminario Tendencias educativas y pedagógicas emergentes desde la perspectiva compleja. . . Barranquilla: Universidad Simón Bolívar.

Oliver, N. B. (2020). The Covid19 Impact Survey: Assessing the pulse of the COVID-19 pandemic in Spain via 24 questions. Accesible . Oliver, N., Barber, X., Roomp, K. y Roomp, K. (2020). The Covid19 Impact Survey: Assessing the pulse of the COVID-19 pandemic in Spain via
24 questions. Accesible en: https://arxiv. org/abs/2004.01014, 10-14 .

Suárez Solana, M. d. ( 2016). Significado externo de "alimentación correcta". Significado externo de "alimentación correcta" en México Salud Colectiva, vol. 12, núm. 4, Universidad Nacional de Lanús Buenos Aires, Argentina, 575-588.

Trenas, A. F. (2012). Análisis y modificación de conducta con los problemas de conducta externalizante. Análisis y modificación de conducta, 38 . 